Brief communication

\title{
Silicon nanowires synthesized by vapor-liquid-solid growth on excimer laser annealed thin gold film
}

\author{
Chao-Yu Meng*, Bo-Lin Shih and Si-Chen Lee \\ Department of Electrical Engineering, Graduate Institute of Electronics Engineering, National Taiwan \\ University, Taipei, 106, Taiwan; *Author for correspondence (E-mail: d89921005@ntu.edu.tw)
}

Received 25 March 2004; accepted in revised form 29 March 2005

Key words: excimer laser annealing, silicon nanowire, gold film, nanoparticle

\begin{abstract}
The fabrication of Si nanowires has been demonstrated using excimer laser annealed thin gold film as the catalyst and vapor-liquid-solid (VLS) growth. Au nanoparticles with mean diameters of 12, 13 and $15 \mathrm{~nm}$ were formed by excimer laser annealing (ELA) of Au film with thickness of 2.5, 5 and $10 \mathrm{~nm}$, respectively. The results show that the silicon nanowires (SiNWs) with desired diameter can be obtained by controlling the Au film thickness and laser power density.
\end{abstract}

\section{Introduction}

In the last few years, nanomaterials have attracted increasing attention due to its quantum size effects and technological interest. Silicon nanowires (SiNWs) in particular are one of the most attractive materials because the existing semiconductor fabrication technologies are mainly based on Si. It can be used as biological and chemical sensors (Cui et al., 2001), field-effect transistors (Chung et al., 2000; Cui et al., 2000), $p$ - $n$ junctions, bipolar transistors, and even invertors (Cui et al., 2001). Different methods such as oxide-assisted catalystfree, and solution technique (Gol et al., 2000; Holmesk et al., 2000; Lee et al., 1999; Marsen \& Sattler 1999; Wang et al., 1999; Zhang et al., 1998), have been developed for SiNWs fabrication, some in bulk quantities. The aim of these works is to produce quantities of nanowires with controlled size. In this paper, we report a new method to synthesize the SiNWs using excimer laser annealed gold film as catalyst, rather than using gold colloids as precursor or tuning ambient pressure to control the diameter of SiNWs. The excimer laser annealing (ELA) combined with vapor-liquid-solid (VLS) method can be easily applied to grow SiNWs with controlled diameter by adjusting the $\mathrm{Au}$ film thickness and laser power density. It is found that the thinner the $\mathrm{Au}$ film as precursor, the more uniform diameter SiNWs can be grown.

\section{Experiments}

One side polished silicon wafers were used as the template in this study. A $200 \mathrm{~nm}$ thick buffered $\mathrm{SiO}_{2}$ were deposited on the substrate by low pressure chemical vapor deposition (LPCVD) method, as shown in Figure 1(a). After deposition, Au film with three different thicknesses, i.e., 2.5, 5 and $10 \mathrm{~nm}$, respectively, were evaporated onto the $\mathrm{Si}$ substrate with e-gun evaporator as shown in Figure 1(b). The $248 \mathrm{~nm} \mathrm{KrF}$ excimer laser with an energy density of $282 \mathrm{~mJ} / \mathrm{cm}^{2}$ was chosen to irradiate the Au film, the laser energy 
(a) Bufferd $\mathrm{SiO}_{2}$

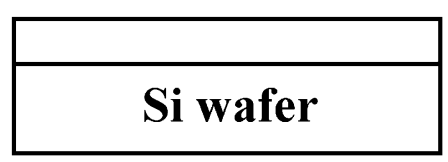

(b)

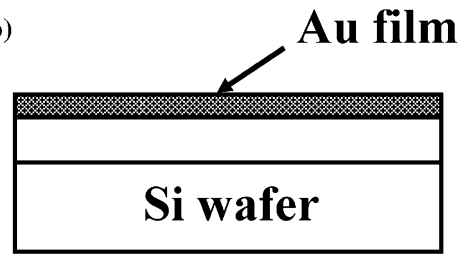

(c)

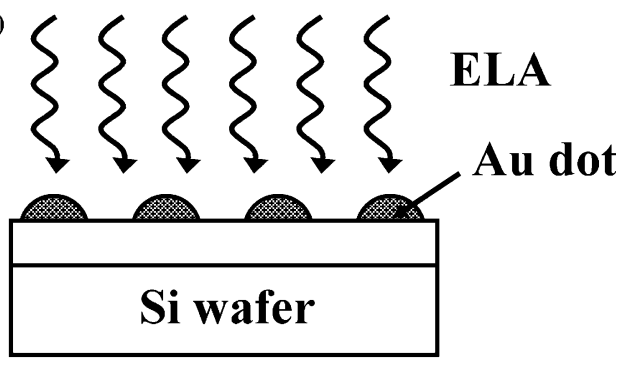

(d)

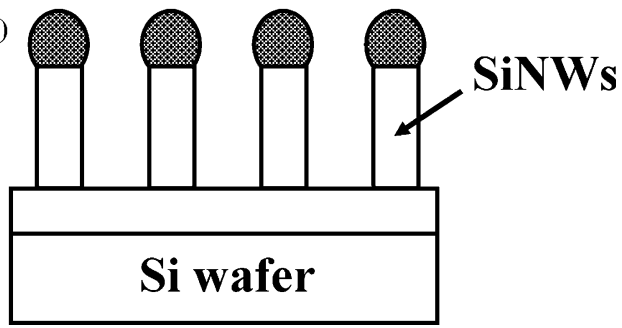

Figure 1. Process flow of the fabrication of Au nanoparticles and SiNWs.

was high enough to totally melt the film and reduce the thermal budget of the substrate due to its short duration (Figure 1(c)). The melted $\mathrm{Au}$ film will randomly condense and form the $\mathrm{Au}$ nanoparticles due to its cohesion force. The VLS growth experiments were then carried out in an isothermal 8-inch diameter cylindrical quartz tube reactor. The Si substrate with Au catalyst on top was placed in a quartz plate, which was situated in the middle of 80 -inch long quartz tube inside the furnace. The system was degassed under vacuum and purged with $\mathrm{N}_{2}$ and then heated to growth temperature at $450^{\circ} \mathrm{C}$. A $10 \%$ diluted $\mathrm{SiH}_{4}$ was then introduced into the tube to initiate the SiNWs growth (Figure 1(d)). The total flow rate was $400 \mathrm{sccm}$ and the total pressure was kept at 30 torr during SiNWs growth.

Planar view images of $\mathrm{Au}$ nanoparticles and SiNWs after VLS growth were performed with a $5 \mathrm{kV}$ LEO 1530 scanning electron microscope (SEM).

\section{Results and discussion}

The effect of the film thickness on the diameters of $\mathrm{Au}$ nanoparticles was first investigated. SEM images of $\mathrm{Au}$ nanoparticles as a function of $\mathrm{Au}$ film thicknesses, i.e., 2.5, 5 and $10 \mathrm{~nm}$, after ELA are shown in Figure 2(a)-(c), respectively. The laser was one shot at $282 \mathrm{~mJ} / \mathrm{cm}^{2}$. The diameter of $\mathrm{Au}$ nanoparticles varied from 6.5 to $26 \mathrm{~nm}$ for $2.5 \mathrm{~nm}$ thick, 6.5 to $48 \mathrm{~nm}$ for $5 \mathrm{~nm}$ thick, and 6.5 to $165 \mathrm{~nm}$ for $10 \mathrm{~nm}$ thick film, respectively. It is found that part of the $\mathrm{Au}$ nanoparticles grow larger when the thickness increases. The reason can be described as follows: first, the excimer laser irradiated from the top of the Au film, the laser power density was chosen sufficiently high to totally melt the entire film. After irradiation, the $\mathrm{Au}$ seeds are randomly formed in the melt as temperature drops. These seeds first condensed will swallow and drain the melted fluid in their neighborhood due to the cohesion force then the seeds will grow up. The later formed seeds become lack of sufficient supply of melt and thus the size is limited. The thicker the film is, the more energy it is absorbed, the longer time and more resource for $\mathrm{Au}$ particles to condense. This also increases the size differences between former and later ones. The average diameter and standard deviation of $\mathrm{Au}$ nanoparticles were calculated from SEM images by statistical analysis. The results were shown in Figure 3, which was obtained by measuring more than 100 nanoparticles from different parts of the sample. The mean diameters of $\mathrm{Au}$ nanoparticles are 12, 13 and $15 \mathrm{~nm}$ for Au film thicknesses of 2.5, 5 and $10 \mathrm{~nm}$, respectively. It is clear that the average nanoparticle diameter increases when the thickness of Au film increases and the variation of size distribution also becomes broader. Therefore, $\mathrm{Au}$ nanoparticles with controlled diameter and smaller size variation can be easily fabricated and obtained by using thinner $\mathrm{Au}$ film thickness. 

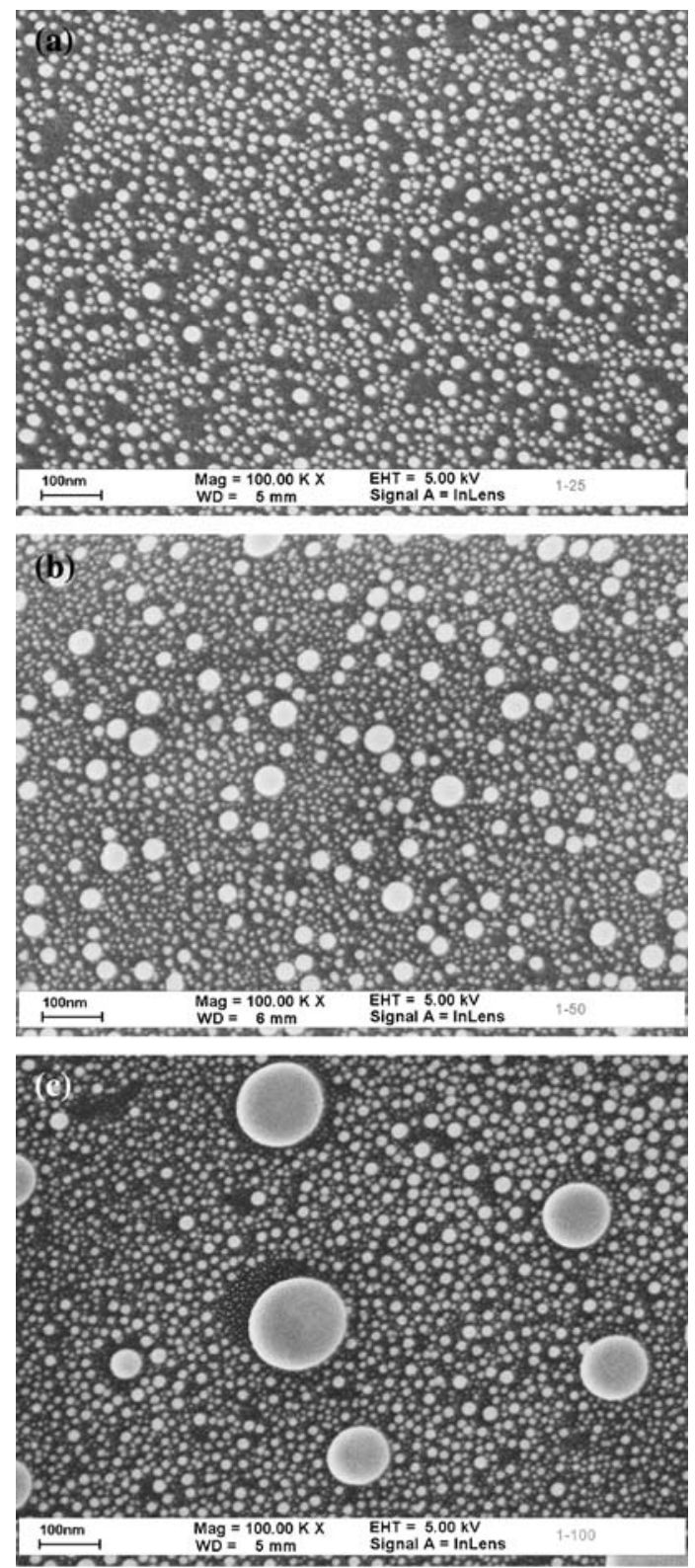

Figure 2. Planar view SEM images of $\mathrm{Au}$ nanoparticles fabricated by ELA on Au films with different thickness, (a) 2.5, (b) 5 and (c) $10 \mathrm{~nm}$.

Figure 4(a)-(c) shows the SEM images which correspond to samples in Figure 2(a)-(c) after VLS growth, respectively. The VLS mechanism for SiNWs growth was first proposed by Wagner and Ellis 1964 (Wagner et al., 1964). In this process, the $\mathrm{Au}$ is used as the catalyst to catalyze the

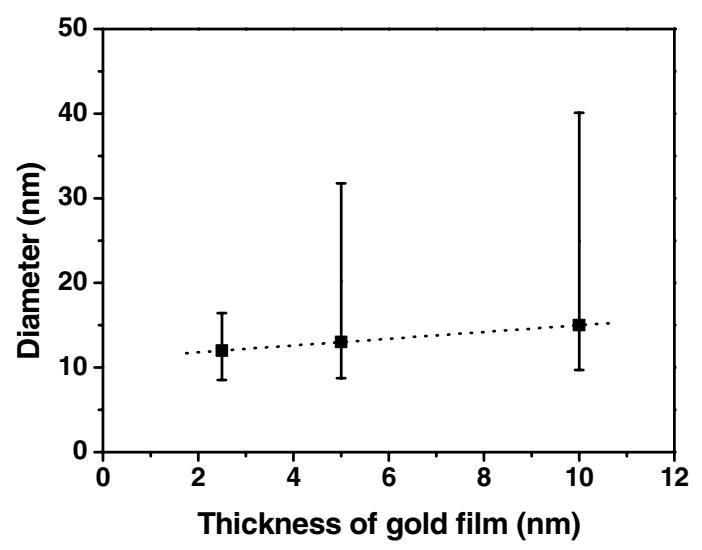

Figure 3. The mean diameter and size variation of $\mathrm{Au}$ nanoparticles as a function of $\mathrm{Au}$ film thickness.

decomposition of $\mathrm{SiH}_{4}$ gas. When the temperature reached the eutectic temperature of $\mathrm{Au} / \mathrm{Si}$ alloy at $363^{\circ} \mathrm{C}$, the decomposition occurred and the $\mathrm{Si}$ atoms started to dissolve into the Au nanoparticles and then form the liquid phase $\mathrm{Au} / \mathrm{Si}$ alloy. Once the concentration of $\mathrm{Si}$ reached its saturation point, the SiNWs began to separate out from the alloy and push it forward at the end. According to this process, the diameter of the nanowire is determined by the original size of the $\mathrm{Au}$ nanoparticles and growth condition. The Au particles at the end of the SiNWs can be clearly observed from Figure 4(a)-(c) and as the direct proof with the VLS growth mechanism. Hence, the diameter of SiNWs is determined by the Au nanoparticles prepared with ELA method. These results demonstrate that the diameter and uniformity of SiNWs can be controlled by determine the Au film thickness.

The diameter of the SiNWs in Figure 4 (a)-(c) varied from 7 to $26 \mathrm{~nm}, 7$ to $51 \mathrm{~nm}$ and 7 to $168 \mathrm{~nm}$, respectively. This result fitted with the variation of $\mathrm{Au}$ nanoparticle diameters after ELA. It is noted that the largest SiNW in Figure 4(c) started to twist and become a wool-like structure. When the diameter of Au nanoparticles is increase, the area of interlayer between SiNWs and liquid phase $\mathrm{Au} / \mathrm{Si}$ alloy is also increase which will increase the defect generation probability. The generated defect in growth will then change the growth direction of SiNWs and thus form the wool like structure. 

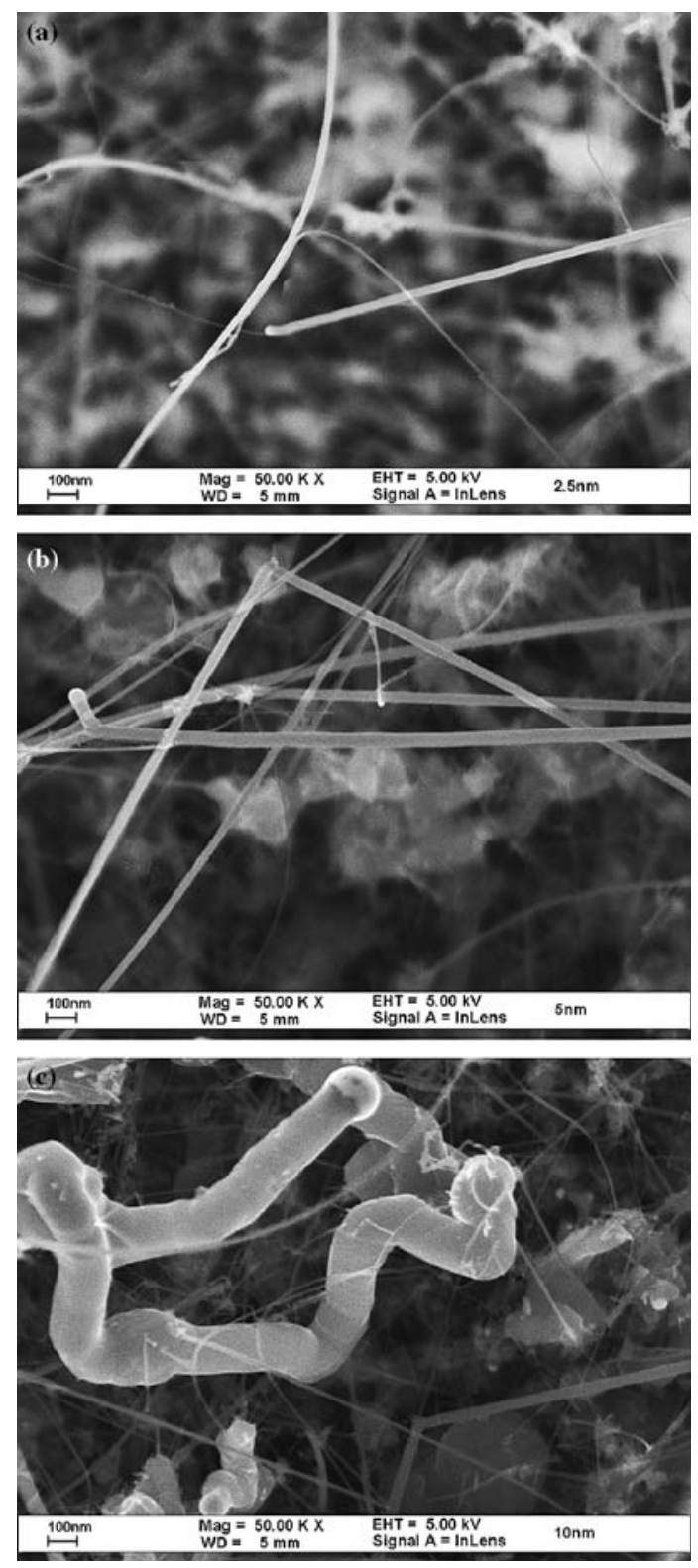

Figure 4. Planar view SEM images of SiNWs after VLS growth on the Au nanoparticles formed by ELA on (a) 2.5, (b) 5 and (c) $10 \mathrm{~nm}$ thick Au film.

\section{Conclusions}

The SiNWs fabricated by ELA combined with VLS method were proposed. The Au nanoparticles fabricated by ELA method with 2.5, 5 and $10 \mathrm{~nm}$ thick $\mathrm{Au}$ film as the precursors had mean diameters of 12,13 and $15 \mathrm{~nm}$, respectively, and the diameter of the nanoparticles varied from 6.5 to $26 \mathrm{~nm}$ for $2.5 \mathrm{~nm}$ thick, 6.5 to $48 \mathrm{~nm}$ for $5 \mathrm{~nm}$ thick, and 6.5 to $165 \mathrm{~nm}$ for $10 \mathrm{~nm}$ thick film, respectively. The size uniformity of the Au nanoparticles can be improved by using thinner $\mathrm{Au}$ film.

\section{Acknowledgement}

This work has been supported by the Industrial Technology Research Institute under Contract No. 092E0090.

\section{References}

Chung S.-W., J.-Y. Yu \& J.R. Heath, 2000. Appl. Phys. Lett. 76, 2068 .

Cui Y., X. Duan, J. Hu \& C.M. Lieber, 2000. J. Phys. Chem. B 104, 5213.

Cui Yi, Q. Wei, H. Park \& C.M. Lieber, 2001. Science 293, 1289.

Cui Y. \& C.M. Lieber, 2001. Science 291, 851.

Gole J.L., J.D. Stout, W.L. Rauch \& Z.L. Wang, 2000. Appl. Phys. Lett. 76, 2346.

Holmesk J.D., K.P. Johnston, R.C. Doty \& B.A. Korgel, 2000. Science 287, 1471.

Lee S.T., Y.F. Zhang, N. Wang, Y.H. Tang, I. Bello, C.S. Lee \& Y.W. Chung, 1999. J. Mater. Res. 14, 4503.

Marsen B. \& K. Sattler, 1999. Phys. Rev. B 60, 11593.

Wang N., Y.H. Tang, Y.F. Zhang, C.S. Lee, I. Bello \& S.T. Lee, 1999. Chem. Phys. Lett. 299, 237.

Wagner R.S. \& W.C. Ellis, 1964. Appl. Phys. Lett. 4, 89.

Wagner R.S., W.C. Ellis, K.A. Jackson \& S.M. Arnold, 1964. J. Appl. Phys. 35, 2993.

Zhang Y.F., Y.H. Tang, N. Wang, D.P. Yu, C.S. Lee, I. Bello \& S.T. Lee, 1998. Appl. Phys. Lett. 72, 1835. 